\title{
Validação de instrumento qualitativo de pesquisa para avaliação das percepções de Fisioterapeutas Oncológicos e estudantes de Graduação em Fisioterapia em relação aos Cuidados Paliativos aplicados em crianças com câncer
}

Validation of a qualitative research instrument to evaluate the perceptions of Oncology Physiotherapists and undergraduate students in Physiotherapy in relation to the Palliative Care applied to children with câncer

Validación de un instrumento de investigación cualitativa para evaluar las percepciones de los fisioterapeutas y estudiantes de pregrado de oncología en fisioterapia en relación con los cuidados paliativos aplicados a niños con cáncer.

Welington Jose Gomes Pereira ${ }^{1 *}$, Cristiane Gonçalves Ribas ${ }^{2}$, Edson Cit Junior ${ }^{3}$, Simone Cristina Pires Domingos ${ }^{3}$, Sonia Aparecida de Almeida ${ }^{3}$.

\section{RESUMO}

Objetivo: Efetuar uma validação de um instrumento qualitativo composto por 20 questões fechadas sobre o tema de Cuidados Paliativos (CP) tenco como objetivo específico avaliar as percepções de Fisioterapeutas e graduandos em Fisioterapia sobre os CP aplicados em crianças com canceres. Método: Neste estudo foram aplicados dois métodos distintos, sendo o primeiro proposto por De Melo WV et al. (2015) e o segundo sugerido por Varanda SS e Benites LC (2017), no processo de validação aplicou-se o conceito de grupos focados, no qual, o primeiro grupo composto por 5 fisioterapeutas especialistas efetuaram avaliação dos seguintes itens: organização, objetividade, clareza, facilidade de leitura e compreensão. Já o um segundo grupo composto de 15 participantes foram submetidos em um teste piloto para avaliar a dinâmica do questionário. Para o cálculo do indice de confiabilidade do constructo foi aplicado o coeficiente de Alfa Cronbach. Resultados: Após o teste piloto o questionário obteve-se um indice de Alfa Cronbach de 0,8 demonstrando que o instrumento de pesquisa possui alta aderencia com os objetivos da pesquisa base. Conclusões: Este formulário qualitativo irá auxiliar a classe científica em pesquisas futuras que visem avaliar as questões éticas, profissionais, emocionais e educacionais de Fisioterapeutas em relação ao tema de Cuidados Paliativos.

Palavras-chave: Validação de questionário, Fisioterapia, Cuidados Paliativos, Câncer.

\section{ABSTRACT}

Objective: To perform a validation of a qualitative instrument composed of 20 closed questions on the topic of Palliative Care (CP) tenco as a specific objective to evaluate the perceptions of Physiotherapists and undergraduate students in Physiotherapy on the CP applied in children with cancers. Method: In this study

\footnotetext{
1'Discente, (PPGEB - Programa de Pós-Graduação em Engenharia Biomédica), Universidade Tecnológica Federal do Paraná (UTFPR), Curitiba/PR.

2 Docente Adjunto do Curso de Fisioterapia, Pontifícia Universidade Católica, Curitiba/PR.

${ }^{2}$ Discente do Curso de Fisioterapia, Centro Universitário Dom Bosco (UniDBSCO), Curitiba/PR.

*E-mail: Welington.pereira@compagas.com.br
} 
two different methods were applied, the first one proposed by De Melo WV et al. (2015) and the second one suggested by Varanda SS and Benites LC (2017), the validation process was applied the concept of focused groups, in which the first group composed of 5 expert physiotherapists evaluated the following items: organization, objectivity, clarity, ease of reading and understanding. A second group of 15 participants were submitted to a pilot test to evaluate the dynamics of the questionnaire. For the calculation of the reliability index of the construct the coefficient of Alfa Cronbach was applied. Results: After the pilot test the questionnaire obtained an alpha Cronbach index of 0.8 demonstrating that the research instrument has high adherence to the objectives of the base study. Conclusions: This qualitative form will help the scientific class in future research aimed at evaluating the ethical, professional, emotional and educational issues of Physiotherapists in relation to the topic of Palliative Care.

Key words: Questionnaire validation, Physiotherapy, Palliative care, Cancer.

\section{RESUMEN}

Objetivo: Realizar una validación de un instrumento cualitativo compuesto por 20 preguntas cerradas sobre el tema de Hospicio (CP) Tenco el objetivo de evaluar las percepciones de los terapeutas y estudiantes en fisioterapia en el CP aplicada en niños con cáncer. Método: En este estudio se aplicaron dos métodos diferentes, el primero propuesto por De Melo WV et al. (2015) y la segunda sugiere balcón y Benites (2017), el proceso de validación aplica el concepto de grupos Siga de discusión en el que el primer grupo de cinco expertos fisioterapeutas a cabo la revisión de los siguientes: organización, con objetividad, claridad , facilidad de lectura y comprensión. Un segundo grupo de 15 participantes se sometió a una prueba piloto para evaluar la dinámica del cuestionario. Para el cálculo del índice de confiabilidad de la construcción se aplicó el coeficiente de Alfa Cronbach. Resultados: Después de la prueba piloto, el cuestionario obtuvo un índice alfa de Cronbach de 0,8 que demuestra que el instrumento de investigación tiene una alta adhesión a los objetivos del estudio base. Conclusiones: Esta forma cualitativa ayudarán a la clase de ciencias en el futuro la investigación dirigida a la evaluación de los fisioterapeutas éticos, profesionales, emocionales y educativas por tema Cuidados paliativos.

Palabras clave: Validación del cuestionario, Fisioterapia, Cuidados Paliativos, Cáncer.

\section{INTRODUÇÃO}

Segundo De Melo WV et al. (2015), a construção e a contextualização de instrumentos de coletas de dados como por exemplo questionários qualitativos são fundamentais para a validação e estruturação de uma pesquisa científica. Para GIL AC (2009), as pesquisas qualitativas que apliquem como método científico a coleta de dados por meio de questionários tem como característica técnica de investigação e o seu propósito é obter informações sobre um determinado assunto, problema ou percepção dos envolvidos, sendo estes instrumentos de pesquisa amplamente utilizados no meio acadêmico e científico.

Para Parasuraman A (1991), os questionários qualitativos devem ter como objetivos aplicarem um conjunto de questões abertas ou fechadas com enfoque de produzir dados específicos que almejem trazer respostas das questões norteadoras e dos objetivos do estudo proposto, devendo estes questionários terem características específicas e estruturadas para não invalidarem as pesquisas científicas.

Conforme De Melo WV et al. (2015), a utilização de questionários em pesquisas científicas não possuem uma metodologia padrão, contendo diversos formatos e métodos distintos aplicados no meio acadêmico, porém, para estes autores todos os questionários devem conter instrumentos avaliativos alinhados diretamente com o escopo da pesquisa independentemente do formato dos questionários a serem aplicados, estas visões destes autores estão congruentes com o ponto de vista apresentado por Parasuraman A (1991).

Ainda segundo De Melo WV et al. (2015), os questionários avaliativos não podem ser confundidos com a metodologia da pesquisa a ser realizada, pois este instrumento avaliativo é uma ferramenta aplicada para 
colher os dados dentro de um estudo e a sua função devem ser vistas como uma engrenagem de um motor que propulsiona $\mathrm{o}$ andamento da pesquisa.

Manzato AJ e Santos AB (2012), descrevem que os levantamentos de dados oriundos de pesquisas qualitativas devem conter cuidados especiais na formulação das suas questões, entre eles, a validade do formato das perguntas e mitigação dos riscos de dubiedade e interpretações equivocadas, estes riscos citados anteriormente podem influenciar as respostas e consequentemente no estudo final. Ainda segundo Manzato AJ e Santos $A B$ (2012), os questionários avaliativos devem ter uma lógica das questões de maneira que a sua representação estejam exatamente alinhadas com os objetivos da pesquisa e possuir uma estrutura de tabulação dos dados específico para mensuração dos resultados, como também uma boa ortografia para facilitar as intepretações dos textos por parte dos entrevistados.

Para Hoss M e Caten CS (2010), uma forma de reduzir estes riscos é efetuar um processo de validação do questionário antes da aplicação deste instrumento em campo, segundo estes autores o processo é chamado de validação interna e tem como objetivo garantir a qualidade e a confiabilidade dos instrumentos de avaliação. Em relação a confiabilidade Hubley AM e Zumbo BD (1996), relatam que a confiabilidade é sinônimo de consistência, estabilidade e previsibilidade, e o uso destes conceitos previamente nos questionários podem garantir uma estrutura de acuracidade, autenticidade e veracidade nos dados coletados.

No estudo efetuado por Haynes SN et al (1995), foi descrito uma outra característica importante no processo de validação interna de questionários, neste caso, os autores relataram a necessidade de validar também os conteúdos das questões a serem aplicadas, pois além dos aspectos de formatação do modelo de questionário é necessário os pesquisadores atentem para as composições das perguntas de modo que sejam garantidos os elementos construtivos principais ,como por exemplo, a coesão e a capacidade de interpretação das perguntas para os entrevistados, deste modo, as perguntas tornam-se mais representativas e relevantes para o objeto de pesquisa. Ainda segundo Haynes SN et al (1995), existem alguns pontos importantes no processo de validação de conteúdo, entre eles: Definir o domínio de construção, geração do conhecimento pretendido, avaliação e correção por especialistas sobre aspectos de ortografia, análises para refinamento dos instrumentos para evitar a dubiedade, entre outras características. Estas etapas relatadas por estes pesquisadores compõem uma estrutura mínima para garantir a boa formulação e organização de um questionário de pesquisa.

Para Manzato AJ e Santos AB (2012) e De Melo WV et al. (2015), os riscos associados com a má formação e estruturação de um questionário avaliativo podem influenciar diretamente uma pesquisa qualitativa, pois objeto de coleta de dados podem conter vícios ou defeitos que comprometem os resultados das avaliações ocasionando distorções dos dados coletados em informações equivocadas e inverídicas que tendem a não corresponder com as opiniões dos entrevistados. Ainda segundo Manzato AJ e Santos AB (2012), para mitigar estes riscos é recomendável uma pré-avaliação e conseguinte aprovação do questionário avaliativo antes de ser aplicado no estudo final, este processo tem como foco efetuar uma pré-verificação se o questionário está apto e conciso para ser utilizado em campo.

Para Hoss M e Caten CS (2010), o processo de validação interna de questionários é aplicado previamente em uma pequena amostra do público alvo devendo este questionário passar por uma averiguação minuciosa para verificar a linguagem aplicada, avaliar e depurar se os conteúdos das questões estão alinhados com objetivos de pesquisa, como também verificar se as perguntas não ferem aspectos éticos e morais dos entrevistados. Esta pré-avaliação é efetuada por um grupo de profissionais do setor ou área da pesquisa com conhecimentos substanciais sobre o assunto. Ainda segundo Hoss M e Caten CS (2010), somente após este processo de validação é possível finalizar o questionário de pesquisa e dar continuidade no estudo nas etapas posteriores.

Manzato AJ e Santos AB (2012), complementam as considerações efetuadas por Hoss M e Caten CS (2010), relatando que após a validação inicial efetuada pelo grupo de especialistas para a formatação do questionário inicial é necessário efetuar uma segunda etapa de teste ou como também conhecido de Teste Piloto. Ainda segundo Manzato AJ e Santos AB (2012), o Teste Piloto é a última fase antes da validação final 
do questionário, pois nesta fase o questionário pré-avaliado é submetido a um grupo de entrevistados que representaram uma amostra do grupo final da pesquisa, esta fase piloto tem como objetivo avaliar possíveis incongruências ou equívocos que tenham passado pelas averiguações efetuadas inicialmente pelos especialistas, como também efetuar uma análise da dinâmica da aplicação do questionário, gerando assim a possibilidade dos pesquisadores ajustarem os pontos ainda latentes do seu estudo de campo. Para Mattar FN (1994), os pré-testes podem ser realizados nos primeiros estágios da formulação dos questionários, pois aplicação destes testes preliminares auxiliam os pesquisadores no delineamento da formulação das questões a serem aplicadas no estudo final.

Segundo o estudo efetuado por Goode WJ e Hatt PK (1972), outra característica positiva do pré-teste é o formato de ensaio geral do experimento onde serão aplicadas as mesmas etapas de entrevista que irá compor no estudo final, simulando aos pesquisadores um ambiente próximo ao que eles iram encontrar no estudo em campo, esta simulação proporcionam aos pesquisadores a possibilidade de avaliarem precocemente as dificuldades ou as necessidades de ajustes nos processos de coletadas de dados.

Frente ao exposto acima, este artigo científico tem como objetivo principal elaborar e validar um questionário qualitativo que será aplicado em grupos focais de Fisioterapeutas especialistas em oncologia e estudantes graduandos de Fisioterapia, tendo como intuito avaliar e mensurar as percepções destes indivíduos sobre os Cuidados Paliativos aplicados em crianças com câncer.

A validação deste questionário partiu da necessidade de alinhar e delimitar as questões propostas do questionário com os objetivos iniciais da pesquisa original, de modo que sejam garantidos que as perguntas do questionário estejam alinhadas com os objetivos específicos do estudo e que estas questões não afetem aspectos éticos ou morais dos entrevistados.

\section{MÉTODOS}

Esta pesquisa foi aprovada pelo comitê de ética do Centro Universitário Dom Bosco (UNIDOM) sob o número CAAE 08799018.6.0000.523 parecer 3.193.355 de 2019, sendo as coletadas de dados efetuados nas dependências da instituição por meio de formulários eletronicos. Os participantes foram convidados para o estudo seguindo os critérios de inclusão e exclusão específicos delimitados por estes pesquisadores, sendo eles:

Para a fase de avaliação inicial por especialistas os participantes devem ser Fisioterapeutas formados com experiência superior a 5 anos na profissão, serem docentes e possuírem a formação acadêmica complementar de pós-graduação de no mínimo Lato sensu de especialização.

Já em relação a seleção para o teste piloto foram adotados os seguintes critérios de seleção: Estudantes (Possuírem idade superior a 18 anos, serem graduandos do curso de Fisioterapia e estarem cursando o $7^{\circ} \mathrm{e}$ 8ํㅜㄹodo do curso, estarem efetuando estágio curricular hospitalar). Fisioterapeutas profissionais (Serem formados em Fisioterapias e terem experiência profissional superior a 2 anos).

Já os critérios de exclusão aplicados neste estudo foram os seguintes: O participante recusar-se para o estudo, não atenderem algum item especificado nos critérios de inclusão. Não assinarem o Termo de Consentimento Livre e Esclarecido (TCLE).

Para o delineamento do escopo do método de pesquisa foi adotado o método proposto por De Melo WV et al. (2015), que estrutura um processo de validação de questionário em 4 etapas, sendo elas:

1 Etapa: Montagem do protocolo e formulário das questões elaboradas sob forma de rascunho preliminar, sendo aplicado a ferramenta de brainstorming por parte dos pesquisadores para a elaboração das perguntas iniciais alinhadas com os objetivos da pesquisa.

2ํㅡㄹapa: Confecção do questionário estruturado com as perguntas elaboradas na etapa anterior, sendo formatado a estética e organização das questões em subtópicos do formulário preliminar. 
3ํㅡㄹ Etapa: Avaliação inicial do questionário por pares pertencentes ao grupo objeto de pesquisa, sendo selecionados estes pares com base em seus conhecimentos substanciais sobre o assunto ou tema.

4 Etapa: Validação interna do questionário proposto, que consiste na execução de um pré-teste em uma pequena amostra da população-alvo, para verificar e analisar os possíveis erros que possam ter passado nas etapas anteriores.

Para a construção das questões avaliativas do questionário foi adotado o roteiro proposto por Aaker DA et al (2001), que organiza em passos lógicos a formulação das questões, conforme apresentado no Quadro 1.

Quadro 1 - Etapas e passos para a formulação das perguntas e estruturação do questionário.

\begin{tabular}{|c|c|}
\hline Etapas & Passos \\
\hline \multirow{4}{*}{$\begin{array}{l}\text { Planejar e avaliar os } \\
\text { itens a serem } \\
\text { pesquisados }\end{array}$} & Especificar os objetivos da pesquisa \\
\hline & Definir o assunto da pesquisa em seu questionário \\
\hline & $\begin{array}{l}\text { Coletar informações adicionais para complementar a sua pesquisa, com base } \\
\text { em literaturas científicas já efetuadas }\end{array}$ \\
\hline & Determinar o que vai ser perguntado aos entrevistados \\
\hline \multirow{2}{*}{$\begin{array}{l}\text { Compor a estrutura do } \\
\text { questionário }\end{array}$} & Definir cada assunto a ser abordado e os conteúdos de cada pergunta \\
\hline & Especificar o formato da pergunta (Aberto ou Fechado) \\
\hline \multirow[b]{2}{*}{$\begin{array}{l}\text { Formatação das } \\
\text { Perguntas }\end{array}$} & Elaborar o formato ortográfico de redação das questões \\
\hline & $\begin{array}{l}\text { Efetuar uma avaliação inicial dos termos aplicados para verificar a facilidade } \\
\text { de compreensão, como também os conhecimentos exigidos para que os } \\
\text { respondentes possam responder com exatidão }\end{array}$ \\
\hline \multirow{2}{*}{$\begin{array}{l}\text { Composição estrutural } \\
\text { do formulário }\end{array}$} & Organizar as questões em ordem estruturada e adequada \\
\hline & Agrupar as questões em subtópicos ou em formato único de questionário \\
\hline \multirow{4}{*}{$\begin{array}{l}\text { Testes e Correção de } \\
\text { Problemas }\end{array}$} & $\begin{array}{l}\text { Efetuar a leitura do questionário inteiro para averiguar possíveis } \\
\text { inconsistências e se ele tem uma lógica das perguntas }\end{array}$ \\
\hline & Verificar possíveis erros de ortografia nas questões \\
\hline & Fazer o pré-teste do questionário entre os pesquisadores \\
\hline & Corrigir os problemas encontrados na fase de pré-teste \\
\hline
\end{tabular}

Fonte: Adaptado de Aaker DA et al (2001).

Para a terceira etapa foi adotado o método proposto por Varanda SS e Benites LC (2017), que especifica que a primeira validação do questionário deve ser efetuada por profissionais especialistas e que detenham conhecimentos substanciais sobre o assunto, sendo recomendado aplicação destas avaliações em grupos focados compostos por pelo menos 5 participantes independentes de sexo, idade, crença. Pois segundo Varanda SS e Benites LC (2017), as opiniões distintas destes especialistas poderão auxiliar os pesquisadores em pontos importantes que não foram abordadas nas etapas anteriores de construção do formulário.

Sendo assim, para a seleção dos participantes especialistas que efetuará a validação do questionário foi aplicado o conceito de amostragem de conveniência, que seleciona os participantes conforme a sua disponibilidade e disposição para participarem da pesquisa, sendo aplicado os critérios de inclusão e exclusão de seleção especificados anteriormente.

Para efetuar a mensuração das avaliações efetuadas pelos especialistas, serão adotados os critérios métricos especificados por Varandas SS e Benites LC (2017), que utilizam uma escala de 0 a 5 pontos, sendo que o score ' 0 ' representa que a questão não contempla os critérios necessários para os objetivos da pesquisa e o score " 5 " nos casos em que as questões satisfazem completamente os critérios necessários para a pesquisa, em cada questão foi destinado um campo em branco para que os entrevistados efetuem sugestões de modificações ou ajustes nas questões conforme as suas interpretações, estas opiniões serão analisadas pelos pesquisadores nos casos de necessidades de ajustes nas perguntas.

Os pontos em avaliação das perguntas em que os especialistas iram avaliar serão os seguintes: (Organização, Objetividade, Clareza, Facilidade de leitura e Compreensão do conteúdo), sendo que cada item será avaliado de 0 a 5 e aceitação da questão pelo grupo será dada pelos seguintes critérios: 
Se a média das avaliações dos especialistas forem superior a $70 \%$ e menores de $90 \%$ será adotado a hipótese que a questão é válida porem necessita de modificações, sendo aplicado as sugestões efetuadas pelos entrevistados.

Nos casos em que as avaliações dos entrevistados possuírem média inferior a $50 \%$ na avaliação final estas questões serão excluídas diretamente do questionário, por fim, nas questões que obtiveram avaliação superior a $90 \%$ nas médias serão mantidas as questões sem modificações do seu formato ou texto original.

O questionário em validação contem 20 questões com características fechadas sendo aplicado a escala Likert de avaliação, as questões foram subdivididas em 4 eixos estruturantes sendo eles: (Conhecimentos Éticos e Morais, Educacional, Profissional e Emocional), tendo como objetivo secundário destas questões avaliar as opiniões destes indivíduos sobre aspectos inerentes a profissão e atividades efetuadas por Fisioterapeutas em equipes multidisciplinares que efetuam Cuidados Paliativos em crianças com câncer.

Entretanto, questionários qualitativos necessitam possuir um nível de confiabilidade de suas questões para garantirem uma consistência para as pesquisas científicas, segundo Cortina JM (1993), um método de análise para estimar o nível de confiabilidade é aplicação de testes estatísticos específicos entre eles destaca-se o coeficiente de Alfa Cronbach desenvolvida pelo pesquisador Lee j. Cronbach no início da década de 1950.

Ainda segundo Cortina JM (1993), o coeficiente de Alfa Cronbach vem sendo aplicado substancialmente nas pesquisas cientificas desde a década de 1950 , tendo a sua aplicação fundamentada e comprovada na literatura, pois permite medir a confiabilidade e o tipo da consistência interna de uma escala, desta maneira é possível avaliar o nível de correlação dos itens de um instrumento de pesquisa.

Para Leontitsis A e Pagge J (2007), para estimar o coeficiente de Alfa Cronbach é necessário elaborar uma matriz onde são quantificadas as respostas do questionário, sendo que a linha no eixo " $X$ " representar o cálculo da somatória das variâncias das respostas dos sujeitos, e no eixo "Y" a somatória das variâncias das respostas de cada questão dos participantes, estes valores irão compor a fórmula de cálculo do coeficiente de Alfa Cronbach.

Desta maneira, de acordo com Leontitsis A e Pagge J (2007), o coeficiente Alfa Cronbach é calculado de acordo com a Equação 1.

Equação 1 - Coeficiente Alfa Cronbach do questionário de pesquisa.

$$
\alpha=\frac{\mathrm{k}}{\mathrm{k}-1}\left[\frac{\sigma_{\tau}^{2}-\sum_{\mathrm{i}=1}^{\mathrm{k}} \sigma_{\mathrm{i}}^{2}}{\sigma_{\mathrm{t}}^{2}}\right]
$$

Segundo Almeida D et al. (2010) o valor mínimo aceitável para o alfa é de 0,70, resultados obtidos abaixo deste índice apresentam uma consistência interna da escala ou questões utilizadas, entretanto, valores acima de 0,90 podem descrever uma redundância ou duplicação nos itens avaliados do mesmo elemento ou constructo, sendo nestes casos preferíveis valores de alfa entre 0,80 e 0,90 de resultados, o que garantem um nível satisfatório de confiabilidade para os questionários.

Para a $4^{\circ}$ etapa do método de validação do questionário foi efetuado um Teste piloto do questionário resultante da 3 etapa, sendo aplicado o roteiro proposto por Varanda SS e Benites LC (2017), sendo selecionado 2 grupos de entrevistados compostos por 7 estudantes de fisioterapia cursado o $7^{\circ}$ e $8^{\circ}$ período do curso de graduação e 8 profissionais Fisioterapeutas formados, seguindo os critérios de inclusão e exclusão adotados no método de pesquisa, o objetivo deste teste piloto é avaliar a dinâmica da aplicação dos questionários e os possíveis erros que possam ter passados nas etapas anteriores. Estes participantes efetuaram o preenchimento das questões conforme a sua opnião nos seguintes campos da escala likert conforme representado na Tabela 1. 
Tabela 1 - Valores da Escala Likert para o teste piloto.

\begin{tabular}{ccc}
\hline Item & Descrição & Valor Estatístico \\
\hline DT & Discordo Totalmente & 1 \\
D & Discordo & 2 \\
I & Indiferente & 3 \\
C & Concordo & 4 \\
CT & Concordo Totalmente & 5 \\
\hline
\end{tabular}

Fonte: Os autores (2019).

\section{RESULTADOS E DISCUSSÃO}

Foram selecionados 5 Fisioterapeutas especialistas para a primeira avaliação do questionário sendo 4 do sexo feminino e 1 do sexo masculino, estes especialistas possuem a titulação mínima de mestrado, sendo identificado que um deles está cursando Doutorado Scritu Sensu. As idades dos respectivos avaliadores têm em média 52,6 $\pm 1,82$ anos, com uma experiência profissional na Fisioterapia em média 29,2 $\pm 1,64$ anos e experiência na área da docência de 21,08 $\pm 3,08$ anos em média, conforme apresentado na Tabela 2.

Tabela 2 - Tabela descritiva dos avaliadores.

\begin{tabular}{ccccc}
\hline Sexo & Idade & Formação & Exp. Docência & Exp. Profissional \\
\hline Feminino & 50 Anos & Mestrado & 20 Anos & 30 Anos \\
Feminino & 53 Anos & Mestrado & 25 Anos & 31 Anos \\
Masculino & 53 Anos & Mestrado & 17 Anos & 28 Anos \\
Feminino & 55 Anos & Mestrado & 20 Anos & 30 Anos \\
Feminino & 52 Anos & Mestrado & 23 Anos & 27 Anos \\
\hline Média & $52,6 \pm 1,82$ & & $21 \pm 3,08$ & $29,2 \pm 1,64$
\end{tabular}

Fonte: Os autores (2019).

Conforme o método de pesquisa, os avaliadores foram convidados formalmente para participarem do estudo, sendo todos orientados referente as premissas relacionadas com objeto de validação, foi solicitado também assinatura do TCLE por parte dos avaliadores, após os devidos esclarecimentos e assinaturas dos TCLE, foi solicitado aos participantes o preenchimento de cada campo de avaliação das questões.

Após o preenchimento das questões com as respectivas avaliações, estes formulários foram entregues para os pesquisadores que efetuaram a tabulação dos dados por meio do software Excel $2016 \AA$, obtendo os seguintes resultados, conforme representado na Tabela 3.

Tabela 3 - Avaliação dos Especialistas do Instrumento de Pesquisa.

\begin{tabular}{|c|c|c|c|c|c|c|c|c|c|c|c|c|c|c|c|c|c|c|c|c|c|c|c|c|c|c|c|}
\hline \multirow{2}{*}{ Questões } & \multicolumn{5}{|c|}{ Organização } & \multicolumn{5}{|c|}{ Objetividade } & \multicolumn{5}{|c|}{ Clareza } & \multicolumn{5}{|c|}{ Leitura } & \multicolumn{5}{|c|}{ Compreensão } & \multirow{2}{*}{ Média } & \multirow{2}{*}{ Situação } \\
\hline & Al & $\mathbf{A 2}$ & A3 & A4 & A5 & Al & A2 & A3 & A4 & A5 & Al & A2 & $\mathbf{A 3}$ & A4 & A5 & Al & $\mathbf{A 2}$ & A3 & A4 & A5 & Al & $\mathbf{A 2}$ & A3 & A4 & A5 & & \\
\hline 1 & 5 & 5 & 5 & 5 & 5 & 5 & 5 & 5 & 5 & 5 & 4 & 5 & 5 & 5 & 5 & 4 & 5 & 5 & 5 & 5 & 5 & 5 & 5 & 4 & 5 & $97,6 \%$ & Ok \\
\hline 2 & 5 & 5 & 5 & 5 & 5 & 5 & 5 & 5 & 5 & 5 & 4 & 5 & 5 & 5 & 5 & 3 & 5 & 5 & 5 & 5 & 5 & 5 & 5 & 5 & 5 & $7,6 \%$ & Ok \\
\hline 3 & 3 & 5 & 5 & 5 & 0 & 4 & 5 & 3 & 4 & 2 & 3 & 5 & 3 & 4 & 5 & 4 & 5 & 5 & 5 & 5 &  & 5 & 5 & 5 & 3 & $2,4 \%$ & Revisão \\
\hline 4 & 3 & 5 & 5 & 5 & 0 & 3 & 5 & 3 & 5 & 5 & 3 & 5 & 3 & 5 & 5 & 4 & 5 & 5 & 5 & 5 & 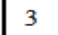 & 5 & 5 & 5 & 5 & $6 \%$ & Revisão \\
\hline 5 & 4 & 5 & 5 & 5 & 4 & 5 & 5 & 5 & 5 & 5 & 5 & $\theta$ & 5 & 4 & 5 & & 5 & 5 & f & 5 & & 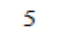 & 5 & 5 & 5 & & Ok \\
\hline 6 & 4 & 5 & 5 & 5 & 5 & 5 & 5 & 5 & 5 & 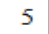 & 3 & 3 & 5 & 3 & 3 & 4 & 2 & 3 & 5 & 5 & 4 & 3 & 5 & 5 & 2 & $\%$ & Ok \\
\hline 7 & 4 & 5 & 5 & 5 & 5 & 5 & 5 & 5 & 5 & 5 & 5 & 5 & 5 & 5 & 5 & 5 & 5 & 5 & 5 & 5 & 5 & 5 & 5 & 5 & 5 & $\%$ & Ok \\
\hline 8 & 4 & 5 & 5 & 5 & 5 & 4 & 5 & 5 & 5 & 5 & 4 & 5 & 5 & 5 & 5 & 3 & 5 & 5 & 5 & 5 & 4 & 5 & 5 & 5 & 5 & $96,8 \%$ & Ok \\
\hline 9 & 4 & 5 & 5 & 5 & 1 & 3 & 5 & 5 & 5 & 1 & 3 & 5 & 5 & 5 & 1 & 4 & 5 & 5 & 5 & 1 & 4 & 5 & 5 & 5 & 1 & $4 \%$ & Revisão \\
\hline 10 & 3 & 4 & 5 & 5 & 1 & 4 & 4 & 5 & 5 & 1 & 4 & 4 & 5 & 5 & 1 & 3 & 4 & 5 & 5 & 2 & 4 & 4 & 5 & 5 & 1 & $2 \%$ & Revisão \\
\hline 11 & 3 & 5 & 5 & 5 & 2 & 3 & 5 & 5 & 5 & 2 & 4 & 5 & 5 & 5 & 2 & 5 & 5 & 5 & 5 & 2 & 3 & 5 & 5 & 5 & 2 & $82,4 \%$ & Revisão \\
\hline 12 & 5 & 5 & 5 & 5 & 5 & 5 & 5 & 5 & 5 & 0 & 5 & 5 & 5 & 5 & 5 & 5 & 5 & 5 & 5 & 5 & 5 & 5 & 5 & 5 & 5 & $0 \%$ & Ok \\
\hline 13 & 3 & 4 & 5 & 5 & 5 & 3 & 4 & 5 & 5 & 5 & 4 & 4 & 5 & 4 & 5 & 3 & 4 & 5 & 5 & 5 & 3 & 4 & 5 & 5 & 5 & $88,0 \%$ & Revisão \\
\hline 14 & 4 & 5 & 5 & 5 & 5 & 4 & 5 & 5 & 5 & 5 & 3 & 5 & 5 & 5 & 5 & 4 & 5 & 5 & 5 & 5 & 4 & 5 & 5 & 5 & 5 & $2 \%$ & Ok \\
\hline 1 & 5 & 5 & 5 & 5 & 5 & 5 & 5 & 5 & 5 & 5 & 4 & 5 & 5 & 5 & $f$ & 5 & 5 & 5 & 5 & 5 & 5 & 5 & 5 & 5 & 5 & & $\mathrm{O}$ \\
\hline 16 & 5 & 5 & 5 & 5 & 1 & 5 & 5 & 5 & 5 & 1 & 5 & 5 & 5 & 5 & 1 & 5 & 5 & 3 & 5 & 1 & 5 & 5 & 5 & 5 & 1 & & Revisão \\
\hline 17 & 3 & 5 & 5 & 5 & 1 & 3 & 5 & 5 & 5 & 1 & 3 & 5 & 5 & 5 & 1 & 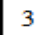 & 5 & 3 & 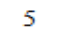 & 1 & 3 & 3 & 3 & 3 & 1 & $0 \%$ & Revisão \\
\hline 18 & 3 & 5 & 5 & 5 & 5 & 2 & 5 & 5 & 5 & 5 & 3 & 5 & 5 & 5 & 5 & 2 & 5 & 5 & 5 & 5 & 3 & 5 & 5 & 5 & 5 & $90,4 \%$ & Ok \\
\hline 19 & 4 & 5 & 3 & 5 & 1 & 5 & 5 & 3 & 5 & 1 & 4 & 5 & 3 & 5 & 1 & 4 & 5 & 3 & 5 & 1 & 4 & 5 & 3 & 5 & 1 & $72,8 \%$ & Revisão \\
\hline 20 & 3 & 5 & 0 & 5 & 4 & 4 & 5 & 0 & 5 & 5 & 3 & 5 & 0 & 4 & 3 & 4 & 5 & 0 & 5 & 5 & 3 & 5 & 0 & 5 & 5 & $72,0 \%$ & Revisão \\
\hline
\end{tabular}

Fonte: Os autores (2019). 
Tabela 4 - Instrumento de pesquisa revisado após avaliação dos especialistas.

\begin{tabular}{|c|c|c|}
\hline Objetivos das perguntas & $\begin{array}{c}\text { Grupos } \\
\text { de } \\
\text { Questões }\end{array}$ & Questões \\
\hline 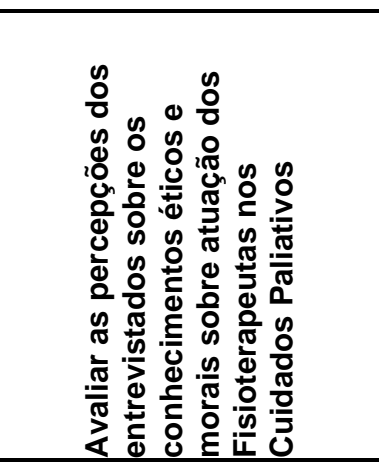 & 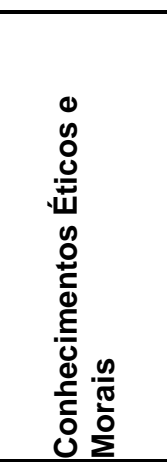 & $\begin{array}{l}\text { 1. Você conhece o conceito e as diferenças entre: Eutanásia, Distanásia e } \\
\text { Ortotanásia? } \\
\text { 2. Você conhece as } 4 \text { fases dos atendimentos aplicados nos cuidados } \\
\text { Paliativos segundo a NA-SNAP (The Australian National Sub-Acute and } \\
\text { Non-Acute Patient Classification) } \\
\text { 3. Você conhece o código de Ética e Deontologia da Fisioterapia } \\
\text { (Resolução COFFITO } 424 / 13 \text { )? } \\
\text { 4. Você concorda com todos os itens especificados no Código de Ética e } \\
\text { Deontologia da Fisioterapia (Resolução COFFITO } 424 / 13 \text { )? } \\
\text { 5. Você já se deparou com alguma questão ética profissional que possam } \\
\text { ter influenciado(a) a sua atuação enquanto estudante ou Fisioterapeuta em } \\
\text { relação aos cuidados paliativos? }\end{array}$ \\
\hline 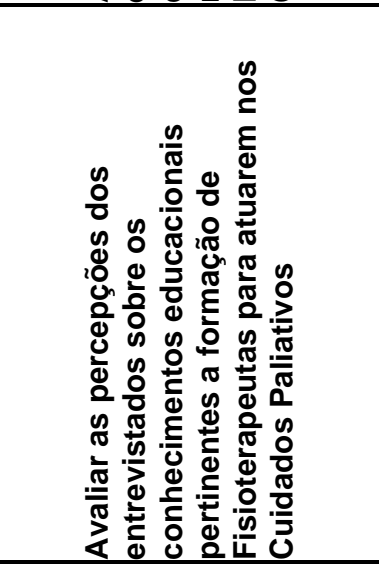 & 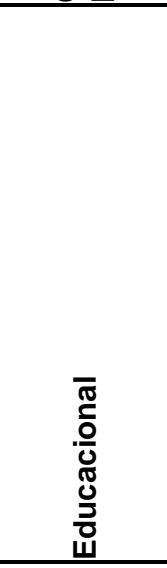 & $\begin{array}{l}\text { 6. Os Fisioterapeutas possuem em sua formação acadêmica } \\
\text { conhecimentos necessários para atuarem nos Cuidados Paliativos } \\
\text { aplicados em crianças com câncer? } \\
\text { 7. Os cursos de graduação em Fisioterapia abordam de forma satisfatória } \\
\text { o tema de Cuidados Paliativos aplicados em criança com câncer? } \\
\text { 8. A carga horária destinada aos Cuidados Paliativos na graduação é } \\
\text { satisfatória para o processo de formação profissional? } \\
\text { 9. Os estudantes de Fisioterapia e Fisioterapeutas profissionais possuem a } \\
\text { cultura de efetuar pesquisas científicas na área de Cuidados Paliativos } \\
\text { aplicados em crianças com Câncer? } \\
\text { 10. Você tem conhecimento a respeito de cursos de capacitação } \\
\text { profissional (Extensão, Pós-Graduação) voltadas para suprir as } \\
\text { necessidades de estudantes ou profissionais Fisioterapeutas em relação } \\
\text { aos Cuidados Paliativos aplicados em crianças com câncer, disponível no } \\
\text { mercado? }\end{array}$ \\
\hline 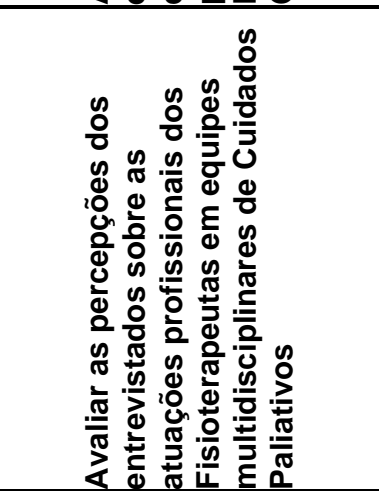 & 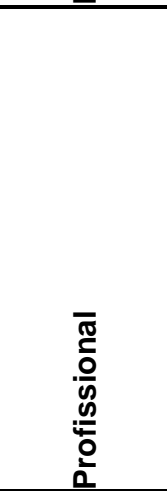 & $\begin{array}{l}\text { 11. Atuação dos Fisioterapeutas é valorizada e reconhecida nas equipes } \\
\text { multidisciplinares de Cuidados Paliativos aplicados em crianças com } \\
\text { câncer? } \\
\text { 12. Você já teve dificuldades técnicas em procedimentos fisioterapêuticos } \\
\text { aplicados em atendimentos de Cuidados Paliativos efetuados em crianças } \\
\text { com câncer? } \\
\text { 13. A formação generalista dos Fisioterapeutas favorece o profissional nos } \\
\text { Cuidados Paliativos aplicados em crianças com câncer? } \\
\text { 14. Os Fisioterapeutas possuem autonomia profissional nas suas atuações } \\
\text { nas equipes multidisciplinares de Cuidados Paliativos efetuados em } \\
\text { crianças com câncer? } \\
\text { 15. Na sua opinião os Fisioterapeutas podem auxiliar na melhora da } \\
\text { qualidade de vida dos pacientes infantis em Cuidados Paliativos? }\end{array}$ \\
\hline 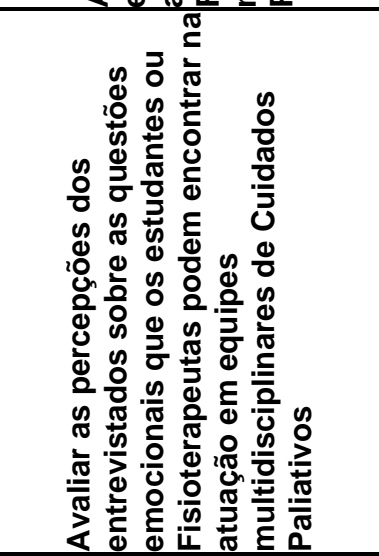 & 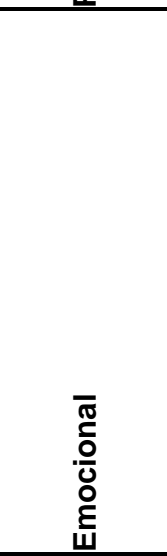 & $\begin{array}{l}\text { 16. Você já teve o sentimento de impotência ou despreparo emocional em } \\
\text { algum atendimento efetuado em Cuidados Paliativos aplicados em crianças } \\
\text { com câncer? } \\
\text { 17. Os Fisioterapeutas e estudantes de Fisioterapia possuem habilidades } \\
\text { técnicas para darem suporte psicológico ou emocional para os seus } \\
\text { pacientes infantis nos Cuidados Paliativos? } \\
\text { 18. Os Cuidados Paliativos em crianças com câncer são mais complexos } \\
\text { do que os efetuados em adultos, no que tange a questão emocional do } \\
\text { profissional? } \\
\text { 19. Os Fisioterapeutas oncológicos estão preparados para trabalhar com } \\
\text { pacientes terminais e lidar com o processo de morte de crianças nos } \\
\text { Cuidados Paliativos? } \\
\text { 20. A morte de crianças com câncer pode afetar o aspecto emocional dos } \\
\text { Fisioterapeutas e estudantes de Fisioterapia na sua atuação em equipes } \\
\text { multidisciplinares de Cuidados Paliativos? }\end{array}$ \\
\hline
\end{tabular}


Fonte: Os Autores (2019).

Segundo as avaliações dos especialistas as seguintes questões: $(1,2,5,6,7,8,12,14,15$ e 18) foram avaliadas com média final superior a $90 \%$ sendo consideradas aptas conforme método de pesquisa proposto, não sendo necessárias alterações destas questões. Entretanto, as questões tiveram avalições superiores a $70 \%$ e inferiores a $90 \%$, deverão passar por um processo de revisão no qual serão aplicados as considerações e recomendações dos especialistas.

As seguintes questões: $(3,4,9,10,11,13,16,17,19$ e 20$)$ foram avaliadas entre $72 \%$ a $88 \%$ de média, por este motivo, estas questões passaram por um processo de revisão e ajustes, tendo como base as recomendações especificadas pelos especialistas. Após o processo de revisão das questões do instrumento de pesquisa, o questionário final foi formatado da seguinte maneira, conforme apresentado na Tabela 4.

Finalizado a etapa de avaliação pelos especialistas, iniciou-se a etapa posterior de teste piloto para verificação da dinâmica de aplicação do questionário como também avaliação do nível de confiabilidade pelo coeficiente de alfa Cronbach. Foram selecionados 15 participantes para a fase do teste piloto, sendo 7 do sexo masculino e 8 femininos, a idade média dos participantes foram de 35,8 $\pm 10,9$ anos, com experiência profissional na área da fisioterapia de $10 \pm 9,07$ anos, possuindo as seguintes formações acadêmicas: Graduandos 33\% ( $n=5)$; Graduados 20\% $(n=3)$; Especialistas 13\% $(n=2)$; Mestres 13\% $(n=2)$; Doutores $20 \%$ $(n=3)$, conforme apresentado na Tabela 5.

Tabela 5 - Tabela descritiva dos avaliadores.

\begin{tabular}{cccc}
\hline Sexo & Idade & Formação acadêmica & Experiência Profissional \\
\hline Masculino & 32 & Graduando em Fisioterapia & 2 \\
Masculino & 55 & Graduado em Fisioterapia & 12 \\
Masculino & 33 & Mestre & 12 \\
Feminino & 26 & Graduando em Fisioterapia & 2 \\
Masculino & 31 & Especialista & 11 \\
Feminino & 23 & Graduando em Fisioterapia & 3 \\
Feminino & 34 & Graduando em Fisioterapia & 2 \\
Masculino & 27 & Graduado em Fisioterapia & 5 \\
Feminino & 28 & Graduando em Fisioterapia & 3 \\
Feminino & 40 & Doutor & 17 \\
Feminino & 31 & Doutor & 6 \\
Feminino & 53 & Mestre & 27 \\
Masculino & 27 & Graduado em Fisioterapia & 3 \\
Masculino & 56 & Doutor & 30 \\
Feminino & 41 & Especialista & 15 \\
\hline Média & $35,8 \pm 10,9$ & & $\pm 9,07$
\end{tabular}

Fonte: Os autores (2019).

Após o preenchimento dos formulários pelos participantes do teste piloto, foram efetuadas as compilações dos dados por meio do software Exce/®2016, como também os cálculos das variâncias das questões e dos sujeitos. A somatória das questões foi computada o valor total de 654 , possuindo a menor avaliação (50) pelo entrevistado E8 e a maior avaliação (84) pelo entrevistado E14, aplicando-se a formula de cálculo da variância das questões foi identificado a somatória de (S1) em 33,33, já para o cálculo da somatória das variâncias dos sujeitos foi identificado o valor de (S2) em 141,5 conforme apresentado na Tabela 6 . Sendo assim, para esta validação do instrumento de pesquisa com base nos valores das variâncias das questões e dos sujeitos, foi efetuado o cálculo do índice de Alfa Cronbach, conforme apresentado na Equação 2.

Equação 2 - Coeficiente Alfa Cronbach do questionário de pesquisa.

$$
\alpha=\frac{\mathrm{k}}{\mathrm{k}-1}\left[\frac{\sigma_{\tau}^{2}-\sum_{\mathrm{i}=1}^{\mathrm{k}} \sigma_{\mathrm{i}}^{2}}{\sigma_{\mathrm{t}}^{2}}\right]=\frac{20}{20-1}\left[\frac{141,54-33,03}{141,54}\right]=0,80
$$


Com base nestes valores obtidos das variâncias foi possível efetuar o cálculo do Coeficiente de Alfa Cronbach, no qual o valor de $k$ representa o número de questões do formulário (20), $S 1=33,33$ e $S 2=141,5$, efetuando aplicação da fórmula em questão obteve-se o valor de confiabilidade do constructo de 0,80 .

Tabela 6 - Resultados do Teste Piloto.

\begin{tabular}{|c|c|c|c|c|c|c|c|c|c|c|c|c|c|c|c|c|c|c|c|c|c|c|}
\hline Sujeito & Q1 & Q2 & Q3 & Q4 & Q5 & Q6 & Q7 & Q8 & Q9 & Q10 & Q11 & Q12 & Q13 & Q14 & Q15 & Q16 & Q17 & Q18 & Q19 & Q20 & Total & S2 \\
\hline E1 & 4 & 4 & 3 & 4 & 3 & 2 & 1 & 2 & 4 & 4 & 5 & 4 & 4 & 4 & 5 & 4 & 1 & 5 & 2 & 5 & 70 & 0,83 \\
\hline E2 & 1 & 1 & 2 & 1 & 4 & 5 & 5 & 3 & 3 & 5 & 5 & 3 & 1 & 5 & 5 & 1 & 1 & 4 & 5 & 5 & 65 & 0,18 \\
\hline E3 & 5 & 4 & 5 & 4 & 2 & 2 & 1 & 1 & 4 & 5 & 4 & 2 & 4 & 5 & 5 & 5 & 1 & 5 & 5 & 5 & 74 & 3,91 \\
\hline E4 & 1 & 1 & 5 & 4 & 1 & 5 & 5 & 4 & 4 & 4 & 4 & 4 & 5 & 4 & 5 & 2 & 5 & 4 & 5 & 4 & 76 & 6,31 \\
\hline E5 & 5 & 5 & 5 & 4 & 5 & 4 & 2 & 2 & 4 & 4 & 4 & 2 & 3 & 1 & 5 & 5 & 4 & 5 & 3 & 5 & 77 & 7,73 \\
\hline E6 & 5 & 2 & 5 & 4 & 2 & 2 & 2 & 2 & 2 & 2 & 2 & 4 & 2 & 4 & 4 & 5 & 2 & 5 & 4 & 5 & 65 & 0,18 \\
\hline E7 & 3 & 4 & 4 & 4 & 4 & 2 & 2 & 2 & 2 & 3 & 2 & 4 & 4 & 2 & 5 & 4 & 4 & 4 & 3 & 4 & 66 & 0,03 \\
\hline E8 & 2 & 1 & 1 & 3 & 4 & 2 & 2 & 2 & 2 & 2 & 2 & 3 & 2 & 4 & 4 & 1 & 2 & 4 & 4 & 3 & 50 & 19,68 \\
\hline E9 & 1 & 5 & 5 & 5 & 4 & 5 & 4 & 4 & 2 & 1 & 2 & 2 & 2 & 4 & 5 & 5 & 1 & 5 & 4 & 5 & 71 & 1,38 \\
\hline E10 & 1 & 1 & 4 & 1 & 5 & 2 & 2 & 2 & 2 & 1 & 1 & 1 & 2 & 1 & 4 & 1 & 1 & 3 & 1 & 4 & 40 & 50,54 \\
\hline E11 & 5 & 1 & 5 & 5 & 1 & 2 & 2 & 2 & 1 & 2 & 2 & 3 & 1 & 2 & 5 & 3 & 2 & 4 & 1 & 4 & 53 & 13,21 \\
\hline E12 & 5 & 5 & 5 & 4 & 5 & 2 & 2 & 2 & 2 & 4 & 4 & 4 & 2 & 4 & 5 & 4 & 2 & 4 & 4 & 4 & 73 & 2,93 \\
\hline E13 & 5 & 5 & 5 & 5 & 5 & 4 & 3 & 2 & 2 & 3 & 4 & 3 & 2 & 5 & 5 & 3 & 4 & 4 & 4 & 4 & 77 & 7,73 \\
\hline E14 & 5 & 5 & 5 & 5 & 5 & 5 & 2 & 2 & 3 & 5 & 5 & 4 & 2 & 3 & 5 & 5 & 5 & 5 & 5 & 3 & 84 & 21,63 \\
\hline E15 & 5 & 1 & 3 & 1 & 3 & 2 & 2 & 3 & 3 & 3 & 3 & 3 & 3 & 3 & 5 & 2 & 3 & 3 & 3 & 4 & 58 & 5,28 \\
\hline Total & 53 & 45 & 62 & 54 & 53 & 46 & 37 & 35 & 40 & 48 & 49 & 46 & 39 & 51 & 72 & 50 & 38 & 64 & 53 & 64 & 654 & 141,54 \\
\hline S1 & 3,3 & 3,4 & 1,7 & 2,1 & 2,1 & 1,9 & 1,6 & 0,7 & 1 & 1,9 & 1,8 & 0,9 & 1,4 & 1,8 & 0,2 & 2,5 & 2,3 & 0,5 & 1,8 & 0,5 & 33,3 & \\
\hline
\end{tabular}

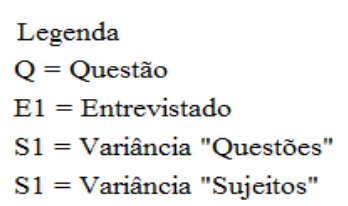

Fonte: Os Autores (2019).

O resultado obtido do índice de confiabilidade do questionário foi de 0,80 , que conforme Streiner DL (2003) é um resultado de alta confiabilidade do nível de correlação das questões do questionário, pois valores abaixo de 0,7 demonstram uma consistência interna de escala considerada baixa e valores acima de 0,9 podem demonstrar um nível de redundância do constructo, neste caso, é preferível valores de coeficiente entre 0,8 e 0,9. Por estes motivos relatados por Streiner DL (2003), o coeficiente para este questionário de pesquisa está dentro dos parâmetros consideráveis para garantir um nível substancial de confiabilidade do instrumento de pesquisa.

\section{CONCLUSÃO}

O papel dos Fisioterapeutas em equipes multidisciplinares de Cuidados Paliativos aplicados em crianças com câncer já é amplamente reconhecido no meio cientifico, pois, estes profissionais possuem habilidades de auxiliar na reabilitação motora, cardiovascular, respiratória e na atenuação das dores, entretanto, existe uma lacuna do conhecimento no que tange os aspectos relacionados com a parte emocional que estes profissionais são submetidos em suas atividades. Por este motivo, a importância da criação de um modelo de instrumento qualitativo que vise buscar respostas para esta lacuna do conhecimento, neste caso, este instrumento de pesquisa validado poderá ser aplicado e auxiliar na obtenção de informações que auxiliem na melhor preparação destes profissionais e consequentemente na melhora dos atendimentos aos pacientes. No processo de validação interna por pares os resultados obtidos pelas avaliações dos especialistas demonstram que o instrumento inicial de pesquisa está de acordo com as premissas e necessidades para futuras pesquisas neste segmento, sendo corroboradas pelo teste piloto efetuado, no qual foi alcançado o índice de confiabilidade pelo teste estatístico de Alfa Cronbach de 0,8. 


\section{REFERÊNCIAS}

1. AAKER DA, et. al. Marketing Research, 7th. John Wiley Operations Research \& Sons, New York, v. 51, n. 4, p. 509$518,2001$.

2. ALMEIDA D, et al. Aplicação do coeficiente alfa de Cronbach nos resultados de um questionário para avaliação de desempenho da saúde pública. XXX Encontro Nacional de Engenharia de Produção, v. 15, p. 1-12, 2010.

3. CORTINA JM. What is coefficient alpha? An examination of theory and applications. Journal of Applied Psychology. v. 78, p. 98-104. 1993.

4. DE MELO WV, et al. Discutindo estratégias para a construção de questionários como ferramenta de pesquisa. Revista Brasileira de Ensino de Ciência e Tecnologia, v. 8, n. 3, 2015.

5. HAYNES SN, et al. Content validity in psychological assessment: a functional approach to concepts and methods. Psychological Assessment, v.7, n.3, p.238-247, 1995.

6. GIL AC. Métodos e técnicas de pesquisa social. 6 $6^{\underline{a}}$ ed. São Paulo: Atlas, 2009.

7. GOODE WJ, HATT PK. Metodos em Pesquisa Social. 4ํo ed. São Paulo: Nacional,1972.

8. HOSS M, CATEN CS. Processo de Validação Interna de um Questionário em uma Survey Research Sobre ISO 9001:2000. Produto \& Produção, v. 11, n. 2, pp. 104-119, 2010.

9. HUBLEY AM, ZUMBO BD. A dialectic on validity: where we have been and where we are going. Journal of General Psychology, v. 23, n.3, p.207-215, 1996.

10. LEONTITSIS A, PAGGE J. A simulation approach on Cronbach's alpha statistical significance. Mathematics and Computers in Simulation. v. 73, p. 336-340. 2007.

11. MANZATO AJ, SANTOS AB. A elaboração de questionários na pesquisa quantitativa. Departamento de Ciência de Computação e Estatística-Universidade de Santa Catarina, 2012.

12. MATTAR FN. Pesquisa de marketing: metodologia, planejamento, execução e análise, 2a. ed. São Paulo: Atlas, 2v., v.2.,1994.

13. PARASURAMAN A. Marketing research. $2^{\circ}$ ed. Addison Wesley Publishing Company, 1991

14. STREINER DL. Being inconsistent about consistency: when coefficient alpha does and doesn't matter. Journal of Personality Assessment. v. 80, p. 217-222. 2003.

15. VARANDA SS, BENITES LC. Validação de instrumentos na pesquisa qualitativa: contribuições de um professor pesquisador em formação. 2017. 